\title{
Fluorescence Lifetime Imaging as an In Situ and Label-Free Readout for the Chemical Composition of Lignin
}

\author{
Sacha Escamez,* Christine Terryn, Madhavi Latha Gandla, Zakiya Yassin, Gerhard Scheepers, \\ Torgny Näsholm, Ola Sundman, Leif J. Jönsson, Judith Lundberg-Felten, Hannele Tuominen, \\ Totte Niittylä, and Gabriel Paës*
}

Cite This: ACS Sustainable Chem. Eng. 2021, 9, 17381-17392

Read Online

S1 Supporting Information

ABSTRACT: Naturally fluorescent polymeric molecules such as collagen, resilin, cutin, suberin, or lignin can serve as renewable sources of bioproducts. Theoretical physics predicts that the fluorescence lifetime of these polymers is related to their chemical composition. We verified this prediction for lignin, a major structural element in plant cell walls that form woody biomass. Lignin is composed of different phenylpropanoid units, and its composition affects its properties, biological functions, and the utilization of wood biomass. We carried out fluorescence lifetime imaging microscopy (FLIM) measurements of wood cell wall lignin in a population of 90 hybrid aspen trees genetically engineered to display differences in cell wall chemistry and structure. We also measured the wood cell wall composition by classical analytical methods in these trees. Using statistical modeling and machine learning algorithms, we identified parameters of fluorescence lifetime that predict the content of S-type and G-type lignin

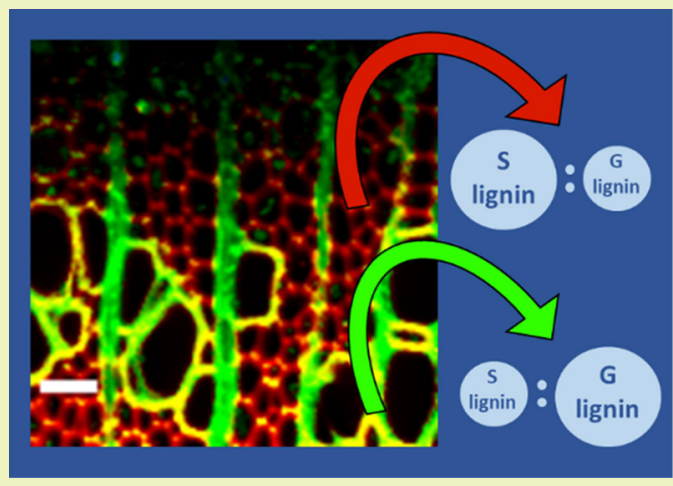
units, the two main types of units in the lignin of angiosperm (flowering) plants.

In a first step toward tailoring lignin biosynthesis toward improvement of woody biomass feedstocks, we show how FLIM can reveal the dynamics of lignin biosynthesis in two different biological contexts, including in vivo while lignin is being synthesized in the walls of living cells.

KEYWORDS: chemotyping in situ, FLIM, lignin, machine learning, statistical modeling, wood

\section{INTRODUCTION}

Woody biomass represents the majority of biomass on land, making it a reservoir of renewable resources for a sustainable economy. This so-called "lignocellulosic" biomass consists of plant cell walls mostly made of three types of natural polymers: cellulose, hemicelluloses, and lignin. ${ }^{2-5}$

Lignin is particularly interesting from both biological and economic points of view. Biologically, lignin influences biochemical and mechanical properties of the cell walls, such as impermeability, ${ }^{6-11}$ rigidity, and mechanical strength. ${ }^{12-14}$ For biorefinery of lignocellulosic biomass, lignin is seen as both a potential source of high-value compounds ${ }^{15,16}$ and one of the major recalcitrance markers to the necessary fractionation of cell wall polymers for different applications. ${ }^{17-19}$

Lignin is produced by polymerization in the cell walls of different types of monomers, mainly hydroxycinnamyl alcohols, which, once integrated as phenylpropanoids into the polymer, are known as G-type, S-type, and, to a lesser extent, H-type lignin units. ${ }^{2,13,20}$ In the wood of flowering trees (angiosperms), the majority of lignin is composed of S-type and G-type units, while lignin can also include atypical units. ${ }^{20}$ Lignin is also made structurally complex by the fact that the same pair of units can form different combinations of covalent bonds between one another. ${ }^{2,13,20}$ The unit composition, as well as the diversity of bonds between units, confers different properties to lignin, ${ }^{2,13,20}$ providing a range of possible mechanical properties to the cell walls. ${ }^{21}$

Interestingly, owing to the molecular structures formed during polymerization, lignin is naturally fluorescent. ${ }^{22-24}$ In theory, fluorescence can be leveraged to measure the chemical composition of lignin in situ, with single-cell-wall resolution. ${ }^{25}$ Several attempts at linking lignin chemistry to fluorescence have relied on monitoring the emission spectrum of lignin. ${ }^{22,23,25}$ These spectral differences in lignin fluorescence have, however, so far rarely been shown to correspond to specific lignin chemical compositions, likely because of technical hinders: the detection of emission spectra can be strongly influenced by interactions, concentration, and external

Received: October 5, 2021

Revised: November 19, 2021

Published: December 11, 2021 
a
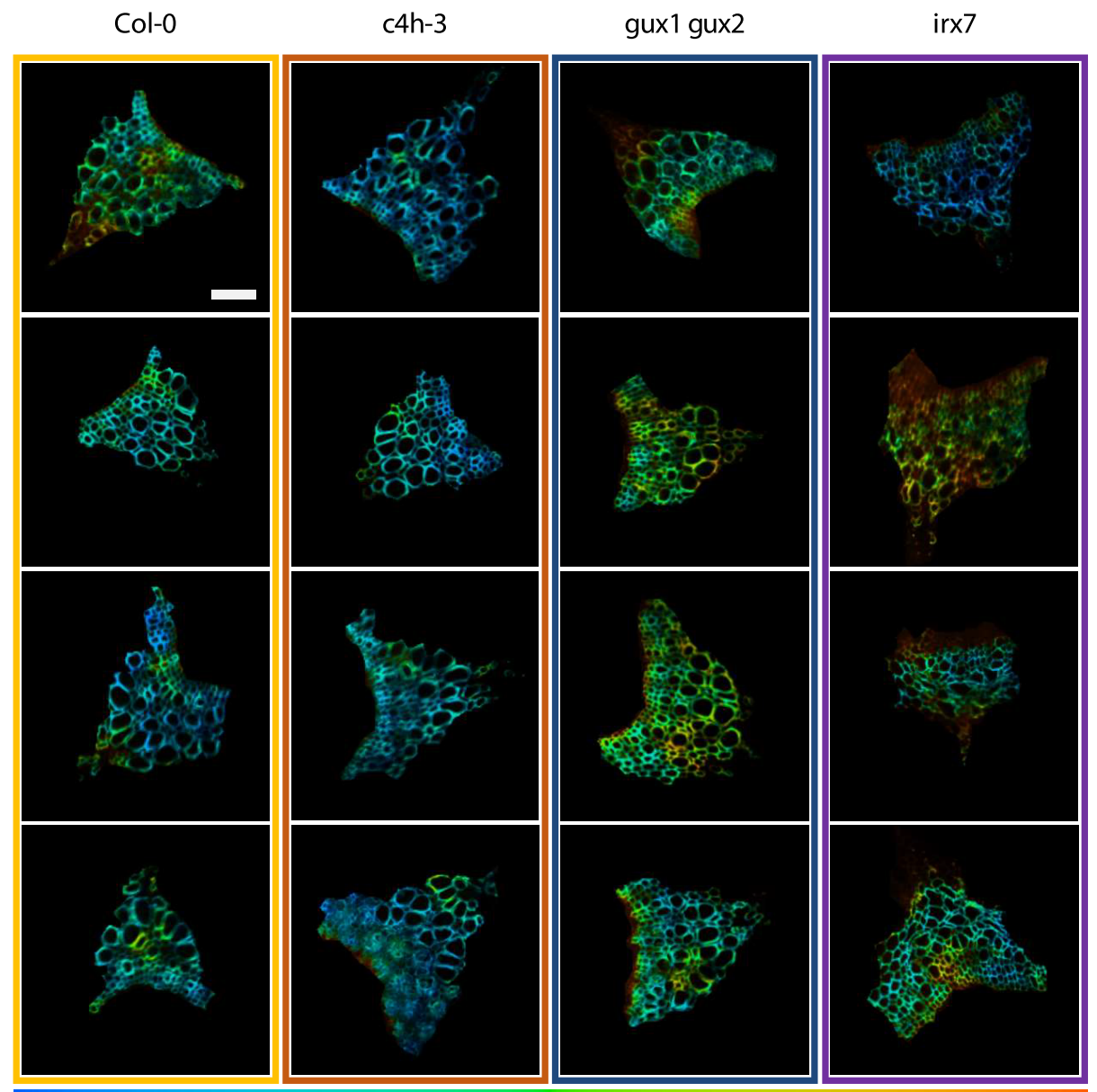

0.55

b

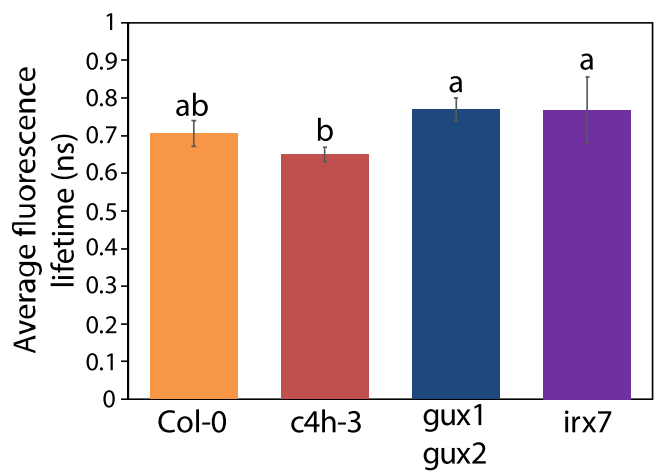

Fluorescence lifetime (ns)

C

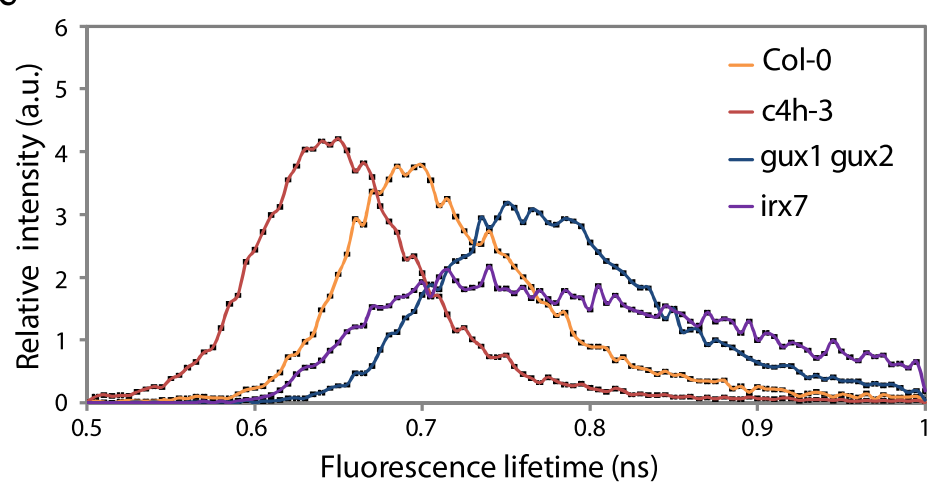

Figure 1. FLIM of Arabidospsis genotypes with different lignocellulosic cell wall properties reveals the potential of FLIM to image cell wall chemistry in situ. (a) Fluorescence lifetime imaging micrographs of a xylem bundle in Arabidopsis thaliana stem cross sections for the indicated genotypes. Xylem cell wall autofluorescence is color-coded based on average fluorescence lifetime for each pixel. Scale bar $=20 \mu \mathrm{m}$. (b) Average xylem cell wall fluorescence lifetime for each Arabidopsis genotype. Error bars represent standard deviation $(n=4$ biological replicates), and genotypes that do not share any letter are significantly different according to a postanalysis of variance (ANOVA) Fisher's least significant difference (LSD) test. (c) Average distribution of xylem cell wall fluorescence lifetime for each genotype $(n=4$ biological replicates).

factors. ${ }^{24,26}$ Another dimension of fluorescence, one that could be correlated more reliably with fluorophore chemical composition, should be leveraged to best exploit the potential of lignin fluorescence as an in situ and label-free chemotyping method.

In line with the theoretical physics that explains atom fluorescence, ${ }^{27}$ Strickler and Berg ${ }^{28}$ derived the equation that provides an account of the fluorescence lifetime of molecules, that is, the time needed for a fluorophore to emit a photon after it has been excited. This so-called Strickler-Berg equation predicts that fluorescence lifetime mostly depends on the chemical composition of the fluorophore, while remaining invariant to many of the factors that affect the emission spectrum, in particular concentration. ${ }^{26}$ The potential of monitoring fluorescence lifetime has more recently been unlocked thanks to the development of advanced pulsed 
lasers $^{29}$ allowing the measurement of fluorescence lifetime between two laser pulses and by recent advances in hardware and methods for photon detection at the nanosecond time scale. $^{29,30}$

Recent studies have started to investigate the potential of using fluorescence lifetime to analyze lignocellulosic cell walls. ${ }^{22}$ Arabidopsis thaliana plants known to display different lignin compositions also showed differences in fluorescence lifetime. ${ }^{31}$ Furthermore, chemical treatment known to affect lignin chemistry resulted in lignin fluorescence lifetime shifts that correlated with treatment severity on the lignocellulosic biomass of several plant species. ${ }^{32,33}$ However, these observations of correlations between lignin fluorescence lifetime and lignin composition did not provide the parameters that would link specific changes in lignin composition with quantifiable variations in fluorescence lifetime.

In this study, we therefore set out to develop fluorescence lifetime as a readout for the lignin chemical composition. To this end, we assembled a population of genetically engineered trees with different wood cell wall properties, such as altered lignin unit compositions. By monitoring wood cell wall chemical composition traits as well as recording parameters of fluorescence lifetime on these trees, we identified fluorescence lifetime parameters that can predict chemical composition traits. We also provided examples of how this new chemotyping method based on fluorescence lifetime imaging microscopy (FLIM) can be used to answer questions of biological and applied relevance. Finally, we discussed how FLIM could be used for in situ chemical composition analyses of other naturally fluorescent polymers.

\section{RESULTS AND DISCUSSION}

Results. Chemically Different Lignocellulosic Cell Walls Display Different Fluorescence Lifetimes. As a proof of concept for the correlation between lignin fluorescence lifetime and the chemical composition of lignocellulosic cell walls, we performed FLIM on xylem tissues in stem cross sections of Arabidopsis thaliana wild-types and mutants previously shown to display modified cell wall chemistry (Figure 1).

The c4h-3 mutation targets an enzyme in the lignin biosynthetic pathway ${ }^{34}$ and had previously been described as triggering an increased ratio of S-type to G-type lignin, ${ }^{35}$ the two main types of lignin in angiosperms, originating from two distinct monomers. ${ }^{2}$ In line with their altered lignin chemical composition, the $c 4 h-3$ mutants showed a downshift in the lignin fluorescence lifetime profile compared to that of Col-0 wild-type plants (Figure 1a-c). The gux1gux2 double mutant is unable to add glucuronic acid and 4-O-methylglucuronic acid (collectively referred to as MeGlcAc) side groups on xylan backbones of hemicelluloses. ${ }^{36,37}$ These MeGlcAc groups of xylan hemicelluloses are thought to covalently associate with lignin. $^{38}$ Their absence in the gux1gux2 mutant should theoretically result in altered lignin fluorescence lifetime: this is confirmed by a shift toward higher lifetime values in guxlgux2 mutants compared with wild-types (Figure 1a-c). Finally, the irx 7 mutant is defective in biosynthesis of xylan hemicelluloses, ${ }^{39}$ which affects the overall cell wall matrix, and, likely indirectly, lignin and its fluorescence lifetime (Figure $1 \mathrm{a}-\mathrm{c})$.

Notably, the genotypes differed not only in terms of average fluorescence lifetime with statistical significance (Figure 1b), but more evidently in the distribution of the observed lifetimes (Figure 1c), with large differences in the maximum wavelength values. In other words, parameters of the fluorescence lifetime appeared as variables that could predict aspects of the lignocellulosic cell wall chemical composition. We therefore set to explore which fluorescence lifetime parameters could be used to predict lignocellulosic cell walls' chemistry.

Genetically Engineered Trees for Cell Wall Chemistry as Standards for FLIM-Based Chemical Readout. To look for predictive correlations between lignocellulosic cell wall fluorescence lifetime and chemical composition, we assembled a collection of hybrid aspen trees (Populus tremula $x$ tremuloides) genetically engineered for modified cell wall properties (Dataset S1). Using Populus trees as a model is justified by the fact that Populus species (poplars, aspens, and hybrids) have been established as model tree species, ${ }^{40}$ with several members having sequenced genomes, ${ }^{41-43}$ and they hold great potential as wood feedstocks. ${ }^{44,45}$

To further increase the variation in cell wall properties, we also submitted these Populus genotypes to two different treatments, consisting of having the majority of their nitrogen nutrition from a mineral or an organic source (Dataset S2). Previously, different amounts of nitrogen fertilization had been shown to alter lignin accumulation in the wood of hybrid aspens. $^{46}$ In another Populus hybrid species, differential nitrogen fertilization resulted in alterations of the wood lignin composition and structure. ${ }^{47} \mathrm{We}$ therefore reasoned that different sources of nitrogen nutrition (with organic nitrogen bearing similarities to low-nitrogen fertilization in terms of tree physiology ${ }^{48}$ ) may add variability to the lignin properties such as abundance of different lignin units or to overall lignin accumulation.

In the resulting collection of 90 trees ( 11 genotypes with two nutrition treatments, including each $4-5$ replicates per genotype and per treatment), we measured 10 traits of the chemical composition of the lignocellulosic cell walls, such as the carbohydrate content (combining cellulose and hemicelluloses), total lignin content, and the proportions of cell wall lignin made of S-type, G-type, and H-type units (Figure 2a; Dataset S3). Furthermore, we measured the release of five different monosaccharides after enzymatic hydrolysis of the cell walls, so-called saccharification (a proxy for biorefinery potential), either without or after a potent acidic pretreatment (Figure 2b; Dataset S3). Finally, we performed FLIM imaging on stem cross sections of the 90 trees (Figure S1) to register 18 parameters of fluorescence lifetime (Figure 2c; Dataset S3).

Nearly all of the traits showed a great variation, with values covering rather evenly the ranges between the mean (set to 0 in scaled and centered values) and the extremes (Figure 2a,c). This variation, and the fact that it is distributed across the full range of values, enables using the measured traits as variables in multivariate analyses and statistical modeling.

Fluorescence Lifetime Parameters Accurately Predict Gtype and S-type Lignin Content. As expected from the wide range of variations in the cell wall chemical composition in the Populus collection, differences in lignin fluorescence lifetime could be detected between genotypes (Figure 3a,b; Figure S1). To predict the cell wall chemical composition, or even saccharification traits, based on fluorescence lifetime parameters, we employed statistical modeling following an established methodology. ${ }^{18,43}$ Briefly, we employed three types of models: linear models, or the more complex machine learning algorithms known as generalized additive models (GAMs) ${ }^{49}$ and Random Forests. ${ }^{50}$ For each cell wall chemical composition trait as well as a selection of relevant 
a
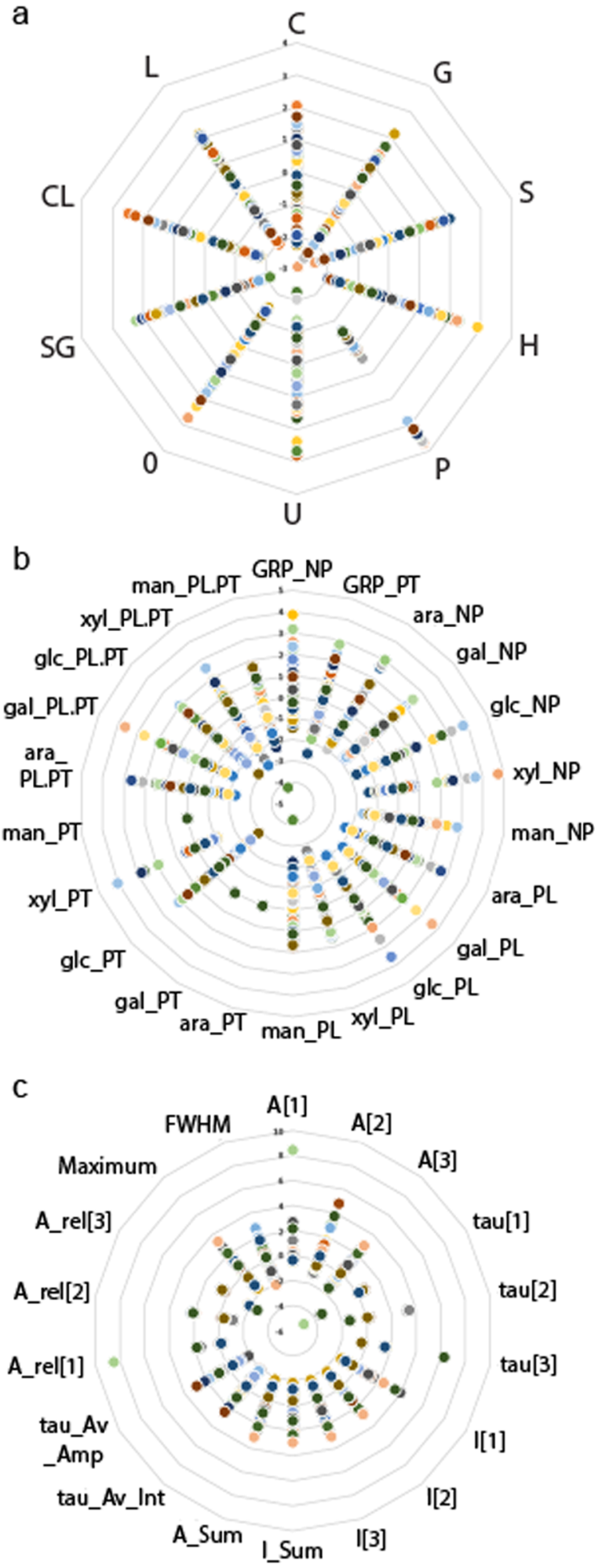

Figure 2. Collection of 90 hybrid aspens displays variation in FLIM parameters, chemical composition, and saccharification, allowing for statistical modeling. (a) Variation in cell wall chemical composition traits (scaled and centered) measured by pyrolysis-gas chromatography/mass spectrometry (GC/MS). Labels indicate the wood content in C: carbohydrates; L: total lignin; G: G-type lignin; S: Stype lignin; $\mathrm{H}$ : H-type lignin; $\mathrm{P}$ : other phenolic lignin units; $\mathrm{U}$ : unidentified compounds; 0: unknown compounds; SG: S-type/Gtype lignin ratio; CL: carbohydrate/lignin ratio. (b) Variation in sugar released from saccharification (scaled and centered) without (NP) or after pretreatment (in pretreatment liquid PL and in hydrolysate PT).
Figure 2. continued

Labels indicate GPR: glucose production rate; ara: arabinose; gal: galactose; glc: glucose; xyl: xylose; man: mannose. (c) Variation in FLIM parameters (scaled and centered). The average lifetime of each image was best described as resulting from three distinct lifetime components (three-exponential fit). Labels indicate $A[n]$ : amplitude for component $[\mathrm{n}]$; tau[n]: average lifetime value for component $[\mathrm{n}]$; $\mathrm{I}[\mathrm{n}]$ : intensity for lifetime component [n]; I_Sum: sum of intensities considering all three components; A_Sum: sum of amplitudes considering all three components; tau Av Int: average lifetime value; tau_Av_Amp: amplitude of the average lifetime; A_rel[n]: relative contribution of component $[\mathrm{n}]$ to the average lifetime.

saccharification traits, we generated at least 15 models of each type, relying on different fluorescence lifetime parameters, to select the best predictive model of each type, as assessed by leave-one-out cross validation (Dataset S4).

While good correlations could be found between fluorescence lifetime parameters and most cell wall traits (Dataset S4), leave-one-out cross validation revealed that only two models could yield accurate predictions $(\mathrm{Q} 2>0.5)$ : the GAMs for G-type and S-type lignin content (Figure 3c,d; Dataset S4), consistent with the fact that the recorded lignin mostly consists of $\mathrm{G}$ and $\mathrm{S}$ units in the wood of Populus trees. Better predictions for the lignin composition were also likely facilitated by the fact that, in addition to using data from our collection of 90 trees, we also included data from lignin samples that either consisted of pure G-lignin or from a synthesized 1:1 mix of S-type and G-type lignin (Dataset S3).

We leveraged these two models to predict the average $S / G$ type lignin ratio for the fibers and for the vessels (Figure $3 e, f$ ) of the developing wood samples of a wild-type tree (Figure 3a) and a tree with reduced expression of the lignin biosynthetic enzyme $\mathrm{C} 4 \mathrm{H}$ ( $c 4 h-\mathrm{RNAi}$; Figure $3 \mathrm{~b}$ ). The average S/G-lignin ratio of the fibers was higher than that of vessels in both trees (Figure 3e,f). The $c 4 h$-RNAi tree (Figure $3 \mathrm{f}$ ) showed a much higher S/G-lignin ratio for both vessels and fibers than in the wild-type tree (Figure 3e). The overall increase in the S/Gtype lignin ratio upon downregulation of $\mathrm{C} 4 \mathrm{H}$ is also consistent with previous observations ${ }^{18}$ and predictions. ${ }^{51,52}$

We sought to investigate whether FLIM parameters could also help predict the frequency of different covalent bonds between lignin units. To this end, we analyzed a subset of 10 wood samples by two-dimensional nuclear magnetic resonance (2D-NMR), which can reveal the frequency of the three most common bonds ${ }^{53}$ between lignin units. ${ }^{54}$ These 2D-NMR measurements (Dataset S4) were compared to the average FLIM parameters measured on the corresponding couples of trees (Dataset S4).

Statistical modeling did not reveal any good predictive model of covalent bond types based on fluorescence lifetime parameters (Dataset S4). However, it cannot be excluded that such predictions could be obtained in the future, as the statistical modeling in this case was strongly constrained by the fact that 2D-NMR analysis only allowed generating 10 observations, instead of the at least 90 observations for the aforementioned chemical composition models. Hence, while future correlative analyses between 2D-NMR and FLIM observations of wood cell walls might yield a FLIM readout for covalent bond types in the lignin polymer, at this point we can only confidently predict S-type and G-type lignin content from fluorescence lifetime parameters. 

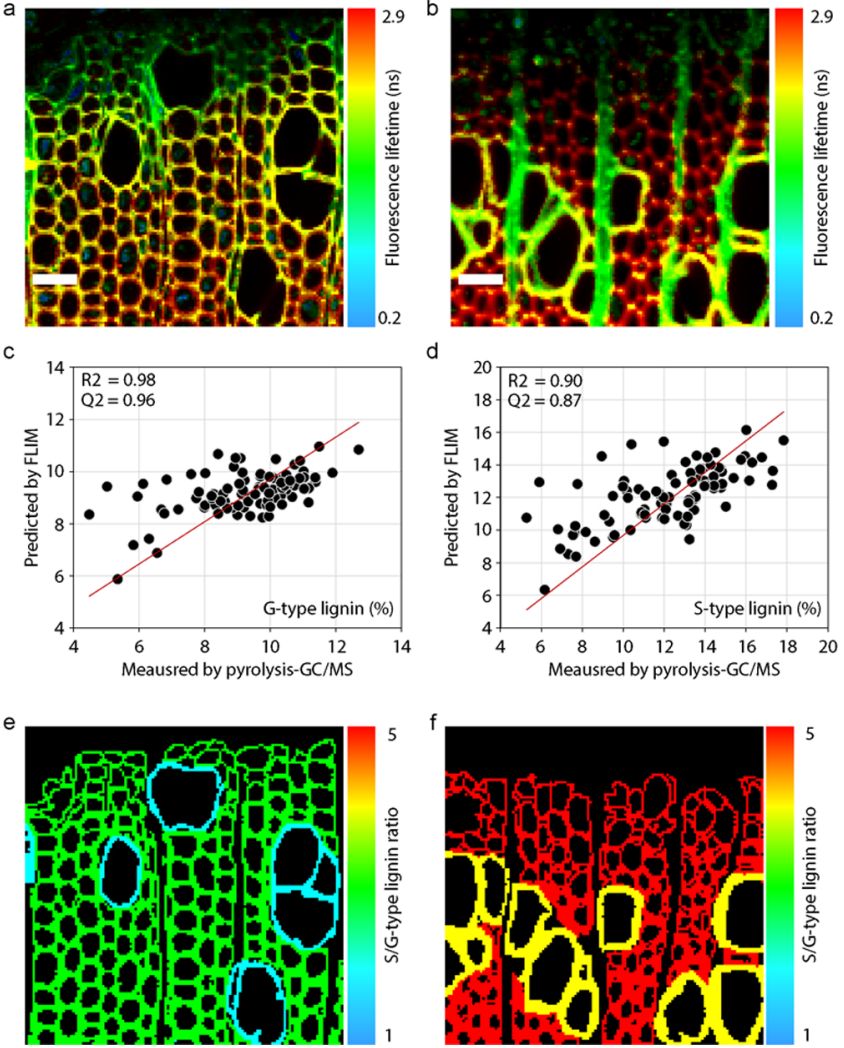

Figure 3. Set of fluorescence lifetime parameters predicts lignocellulosic cell wall S-type lignin and G-type lignin content in situ. (a) Fluorescence lifetime imaging micrograph of wild-type developing wood (directly next to the cambium, the nonfluorescent area at the top of the image). Scale bar $=30 \mu \mathrm{m}$. (b) Fluorescence lifetime imaging micrograph of $c 4 h$-RNAi (2081-B) developing wood section (directly next to the cambium, the nonfluorescent area at the top of the image). Scale bar $=30 \mu \mathrm{m}$. (c) Scatter plot of the G-lignin content, measured versus predicted with the best statistical model (Dataset S4). R2 indicates the fraction of the variance that is explained by the model, while Q2 evaluates the predictivity of the model ( $1 \leq \mathrm{Q} 2<-\infty$, with 1 representing a perfect prediction). (d) Scatter plot of the S-lignin content, measured versus predicted with the best statistical model (Dataset S4). R2 indicates the fraction of the variance that is explained by the model, while Q2 evaluates the predictivity of the model $(1 \leq \mathrm{Q} 2<-\infty$, with 1 representing a perfect prediction). (e) Micrograph of wild-type developing wood (from (a)), where the vessels on the one hand, and the fibers on the other hand, were pseudocolored based on the average $S / G$ ratio for this cell type, as predicted in each sample by the statistical models for S-type and G-type lignin content ((c), (d), and Dataset S4). (f) Micrograph of $c 4 h$-RNAi (2081-B) developing wood (from (b)), where the vessels on the one hand, and the fibers on the other hand, were pseudocolored based on the average $S / G$ ratio for this cell type, as predicted in each sample by the statistical models for S-type and Gtype lignin content ((c), (d), and Dataset S4).

FLIM on Live Xylem Cells In Vivo Reveals the Dynamics of Lignin Deposition in Arabidopsis Roots. FLIM should be applicable in live cell imaging as the FLIM instrumentation consists of a laser confocal microscope base. Hence, we set up to image the first root xylem vessel cells that start depositing lignin in the protoxylem cell files of living Arabidopsis thaliana seedlings. Previous studies in Arabidopsis seedlings' roots had reported that lignin occurred both in the walls of xylem vessel cells and of endodermal cells (forming the Casparian strip). ${ }^{55,56}$ The combined lignin from these two tissues was measured as a bulk and found to consist of the canonical Gtype, H-type, and S-type units, ${ }^{55,56}$ suggesting that FLIM imaging of developing xylem vessel cells in root tips should allow quantification of S-type and G-type lignin content on the walls of these cells.

It had been previously proposed that lignification dynamics would result in G-type lignin being accumulated comparatively earlier than S-type lignin. ${ }^{57-59}$ For the first time, this hypothesis can be verified by live cell imaging thanks to our finding that FLIM parameters can be used to estimate S-type and G-type lignin.

In wild-type seedlings, the ratio of S-type to G-type lignin increased within $6 \mathrm{~h}$ from the first observations (Figure $4 \mathrm{a}, \mathrm{b}$ ), consistent with previous hypotheses about lignification dynamics. ${ }^{58,59}$ In contrast, the $c 4 h-3$ mutants, ${ }^{34}$ which display reduced expression of one of the first enzymes in the biosynthesis of lignin monomers (all types), showed a high S/G-lignin ratio early, with a decrease after $6 \mathrm{~h}$ (Figure 4a,b).

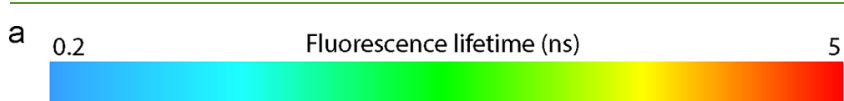

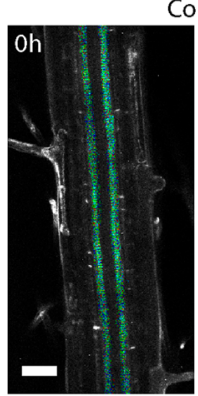

Col-0
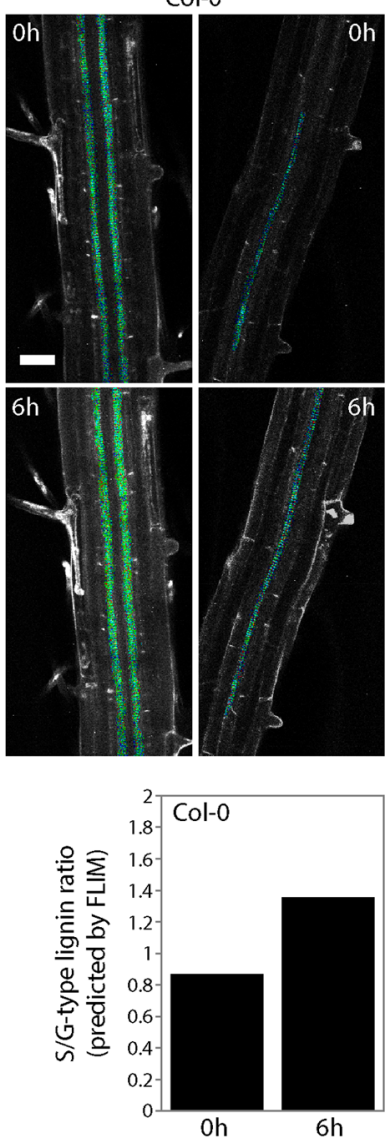

$4 h-3$
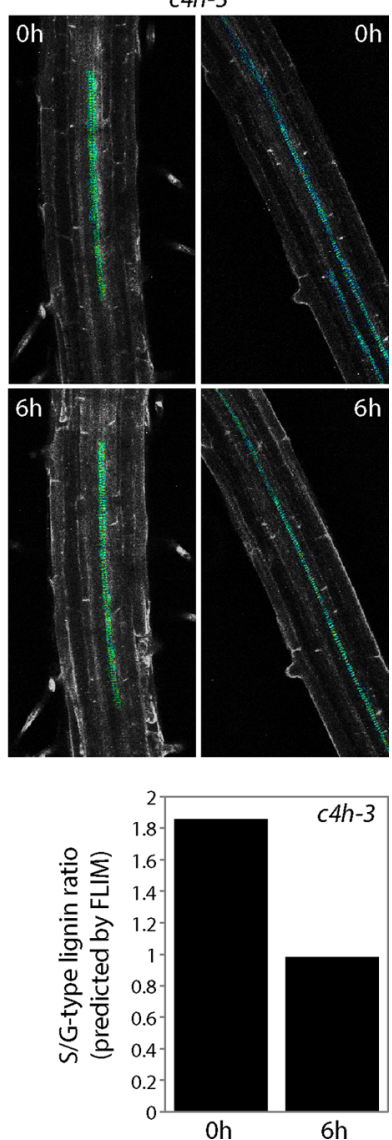

Figure 4. Live imaging of differentiating xylem cells reveals dynamic changes in lignin composition over the course of xylem cell wall biosynthesis. (a) Fluorescence lifetime micrographs of the first lignifying protoxylem tracheary element cells. The color indicates the average lifetime of the cell wall autofluorescence. Scale bar $=40 \mu \mathrm{m}$. (b) Average value of the S/G-lignin ratio for each genotype (as in (a)), as predicted from FLIM parameters using the best statistical models for G-type and S-type lignin (Dataset S4). These average values are from the two different seedlings (in (a)) observed over time in independent experiments. 


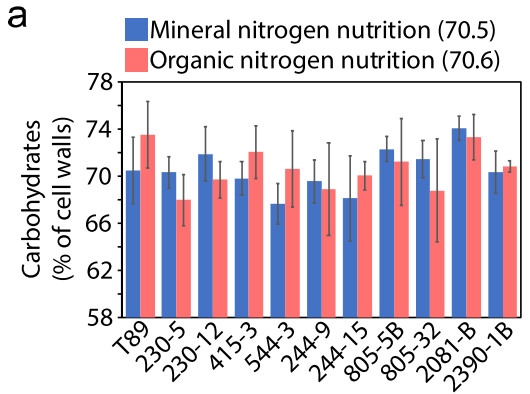

d
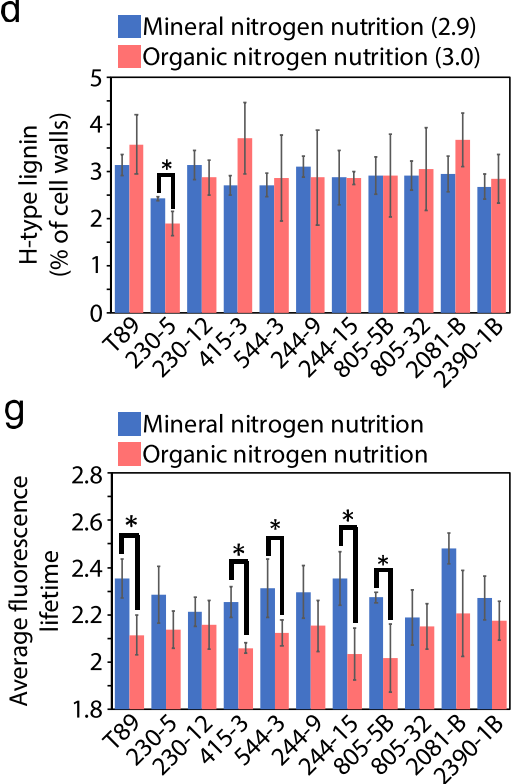

b

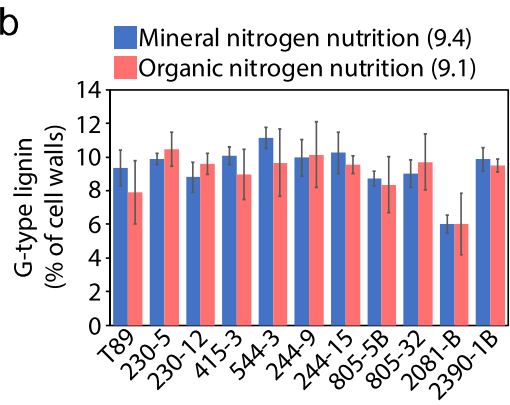

e

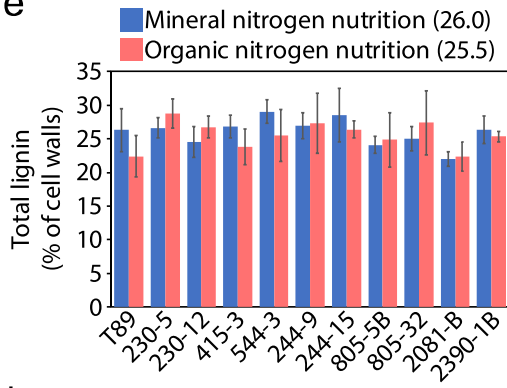

h

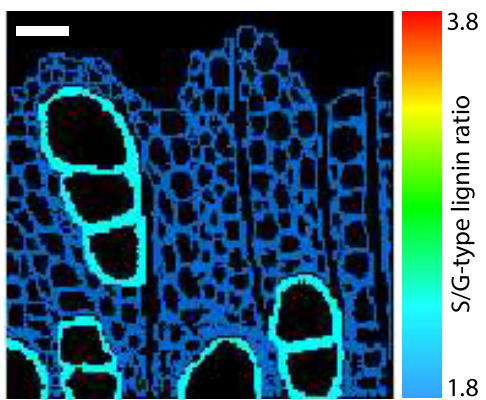

C
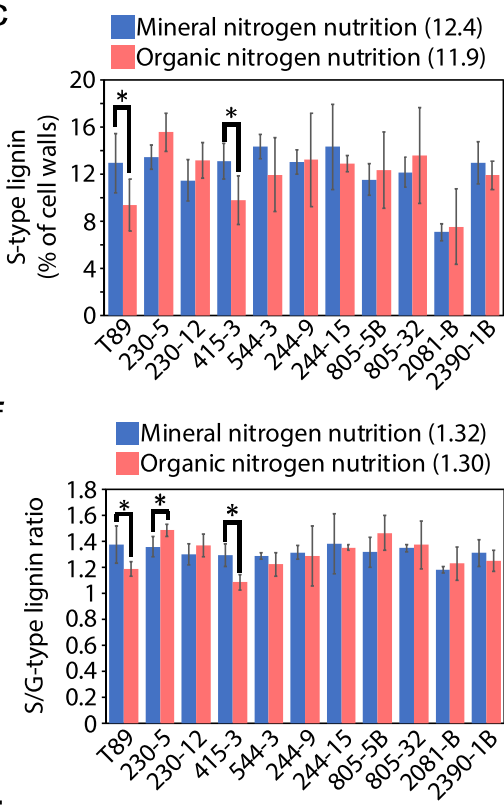

i

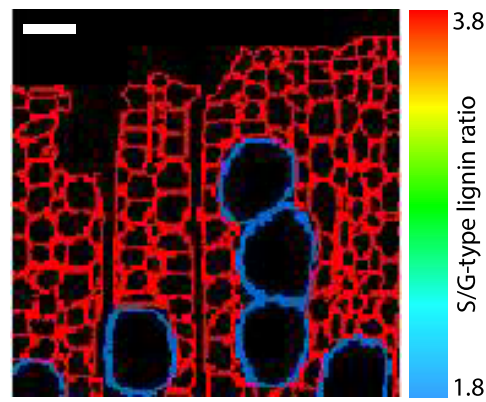

Figure 5. Effect of organic and mineral nitrogen fertilization on lignin chemical composition and lignification dynamics. (a-g) Average value for each genotype under organic (red) versus mineral (blue) nitrogen nutrition, for cell wall contents of carbohydrates (a), G-lignin (b), S-lignin (c), $\mathrm{H}$-lignin (d), and total lignin (e), as well as S-type to G-type lignin ratio (f) and cell wall fluorescence lifetime (g). Error bars indicate standard deviation. Asterisks indicate significant differences $(p<0.05)$ according to a post-ANOVA Fisher LSD test. (h and i) Micrographs of wild-type developing wood under organic $(h)$ or mineral (i) nitrogen nutrition, where the vessels on the one hand, and the fibers on the other hand, were pseudocolored based on the average S/G-lignin ratio for this cell type, as predicted in each sample by the statistical models for S-type and G-type lignin content (Figure 3c,d and Dataset S4). Average values for the S/G-lignin ratio were calculated (for each cell type) from three biological replicates for each nutrition treatment. Scale bar $=30 \mu \mathrm{m}$.

FLIM Reveals Differences in Developing Cell Wall Lignin Composition in Response to Tree Nutrition. Our use of two fertilization treatments during tree growth allows us to investigate whether these different fertilizations could lead to differences in wood chemical composition. Based on our massspectrometry analyses of homogenized wood, which reflect the average chemical composition across the wood (i.e., not capturing potential local, in situ heterogeneity), we could not observe any consistent difference in wood chemical composition matching the different fertilization treatments (Figure 5af). There are, however, notable exceptions to this trend: for two genotypes, the trees having received mineral nitrogen showed higher S-lignin content than the trees having received organic nitrogen (Figure 5c). For another genotype, a higher H-type lignin content was observed under mineral nitrogen nutrition as compared to organic nitrogen nutrition (Figure $5 \mathrm{~d}$ ). These three genotypes also displayed a different S/G-type lignin ratio under the different types of nutrition (Figure $5 f$ ). For all other genotypes, there was no detectable significant difference in lignin content or composition between mineral and organic nitrogen nutrition (Figure $5 \mathrm{a}-\mathrm{f}$ ).
In contrast with the average wood chemical composition, the average fluorescence lifetime of the imaged wood section showed a consistent trend across genotypes, with higher fluorescence lifetime values in trees having received mineral nitrogen rather than organic nitrogen (Figure $5 \mathrm{~g}$ ). This clear trend suggests that while no chemical differences could be detected in homogenized wood samples, there could be greater heterogeneity in the wood chemical composition that may be picked up by FLIM.

FLIM observations of specific areas of developing wood, where lignification is ongoing and therefore more dynamic, did reveal differences in the lignin composition between wild-type trees treated with organic nitrogen (Figure $5 \mathrm{~h}$ ) and mineral nitrogen (Figure 5i). Based on predictions of S-type and Gtype lignin content from FLIM images, the cell walls of fibers and vessels had on average a similar S/G-type lignin ratio under organic nitrogen fertilization (Figure $5 \mathrm{~h}$ ). Under mineral nitrogen fertilization, vessels had on average a much lower S/ G-type lignin ratio than fibers (Figure 5i). The vessels of trees fertilized with mineral nitrogen (Figure 5i) and both fibers and vessels of trees fertilized with organic nitrogen (Figure $5 \mathrm{~h}$ ) 
showed comparable S/G-lignin ratios. Hence, the main difference between the lignin deposited under these fertilization conditions consists of a relative enrichment in S-type lignin (roughly $100 \%$ more) in the fiber cell type upon mineral nitrogen nutrition.

\section{DISCUSSION}

Previous studies had reported shifts in fluorescence lifetime of lignocellulosic cell walls, either as a result of genetic modification that targets lignin biosynthesis ${ }^{31}$ or as a result of chemical treatments altering the chemical and structural environment of lignin. ${ }^{32,33}$ Similarly, we observed shifts in fluorescence lifetime when observing a larger sample set of Arabidopsis thaliana mutants affected in different aspects of lignocellulosic cell wall composition (Figure $1 \mathrm{a}-\mathrm{c}$ ). However, these observations also revealed that the relationship between the cell wall chemical composition and the average fluorescence lifetime of a sample is not linear (Figure $1 \mathrm{~b}$ ). This is consistent with the fact that lignin is a complex fluorophore and implies that several parameters of fluorescence lifetime should be considered to read out the lignocellulose cell wall chemical composition from FLIM.

Next, we assembled a set of 90 trees from 11 genotypes, selected for their expected changes in cell wall properties, to serve as standards to identify relationships that predict the lignocellulosic cell wall chemical composition based on FLIM. We observed multiple cell wall compositions and saccharification traits (Figure 2a,b; Dataset S3), as well as multiple parameters of fluorescence lifetime measurements (Figure 2c; Dataset S3), leading us to find FLIM parameters that could accurately predict either G-type lignin content (Figure 3c; Dataset S4) or S-type lignin content (Figure 3d, Dataset S4). The fact that G-type and S-type lignin can be predicted from FLIM is consistent with the fact that lignin is responsible for the fluorescence of lignocellulosic cell walls. ${ }^{22,24}$ It is also consistent with the fact that in flowering trees most of the lignin in the xylem is composed of S-type and G-type units, ${ }^{2}$ with xylem vessel cells differentiating earlier and usually displaying a higher proportion of G-type units in their lignin compared to fibers.

S-type and G-type lignins are made of aromatic molecules which have a very strong autofluorescence. Imaging plant cell walls through lignin autofluorescence has a long history, ${ }^{24}$ but it rarely allowed discriminating S-type and G-type units. Measuring fluorescence lifetime is much more sensitive because it is based on the evaluation of the way electrons circulate in the aromatic ring after excitation by photon absorption. Even a small molecular difference in the unit structure, such as the additional methoxy group in S-type compared to G-type units, ${ }^{2}$ will change the aromaticity of the ring and lead to shifts in electron circulation and subsequently in fluorescence lifetime.

A small percentage of lignin can consist of atypical units such as those derived from monolignol biosynthetic intermediates. $^{20,60,61}$ Lignin often also comprises a small proportion of conjugated units, whereby classical units bear additional chemical groups. ${ }^{20,62}$ Our calibration dataset recorded only S-type, G-type, H-type, and "other phenolics" lignin units (Dataset S3), without the much more technically challenging quantification of specific atypical or conjugated lignin units. While it is likely that atypical lignin units would have been accounted for in our analyses as "other phenolics," we cannot exclude that the analytical methods we used would misclassify some atypical or conjugated lignin units as, for example, S-type or G-type units. Such hypothetical misclassification would only affect the FLIM-based measurements of S-type and G-type units by a few percent, which means that the conclusions we draw in this article would remain valid. Yet, future work could use plant genotypes genetically designed to synthesize lignin highly enriched in atypical or conjugated units as standards to enable the detection and quantification of such atypical lignin units by FLIM. The work we present here only lays the foundation for the development of FLIM as a chemical readout on lignocellulosic cell walls, and the possibilities virtually encompass the detection and quantification of any type of lignin units by FLIM in the future.

Other cell wall composition traits or saccharification traits could not be predicted by FLIM in our dataset. The question remains of whether future work could identify a relationship, or if there is no relationship to be found. For example, complex, composite traits such as release of sugars from saccharification might depend on many other traits, ${ }^{18}$ only a fraction of which might be related to lignin fluorescence lifetime. In fact, our failure to predict saccharification traits based on FLIM parameters is consistent with the theoretical physics of fluorescence lifetime. The theory indeed implies that fluorescence lifetime is only tied to the chemical composition of the fluorophore. ${ }^{2,28}$ Leaning on this theoretical basis, fluorescence lifetime parameters measured on lignocellulosic cell walls should be expected to only relate to the fluorophore lignin and to other molecules covalently binding to it (e.g., hemicelluloses, consistent with results shown in Figure $1 \mathrm{a}-\mathrm{c}$ ), but not to more loosely related traits such as sugar release from saccharification.

Based on the same theoretical physics background, FLIM may also display a relationship to the frequency of different covalent bonds between units in the lignin polymer, but we failed to identify such relationships (Dataset S4). A likely explanation resides in the number of observations, as we could only quantify covalent bonds between lignin units in a set of 10 samples (while all other traits could be measured on at least 90 samples). Future studies with a greater number of samples may reveal relationships between FLIM parameters and frequencies of different covalent bonds within lignin.

On the other hand, variations in fluorescence lifetime might be specifically linked to the aromatic properties of the fluorophore. Covalent bonds connecting these aromatics are intrinsically not fluorescent. One could have expected that different bonds would lead to different FLIM signals, but given the variety of bonds and the influence of the environment (namely polysaccharides around lignin), it may be that lignin covalent bonds cannot be distinguished accurately by FLIM within the cell wall matrix.

Our discovery of models capable of predicting G-type and Stype lignin from FLIM enabled us to monitor the dynamics of lignin deposition in the walls of developing xylem cells in vivo, thanks to the fact that FLIM measurements are compatible with live cell imaging. Looking at the first lignifying xylem tracheary element cells in the root of Arabidopsis thaliana seedlings, we could visualize a shift in the ratio of S-type to Gtype lignin. Our direct, in vivo observations revealed that more G-type lignin is deposited early followed by an enrichment in S-type lignin over the next $6 \mathrm{~h}$ in wild-type plants (Figure $4 a, b)$. This is in line with previous, indirect observations which had raised the question as to whether G-lignin would be mostly deposited earlier than S-lignin. ${ }^{57-59}$ 
The dynamics of lignification were different between wildtype plants and the $c 4 h-3$ mutant genotype (Figure $4 a, b$ ), whose mutation is known to affect lignin monomer biosynthesis in the cytoplasm. Observing differences in the dynamics of lignification between these genotypes is therefore in line with the idea that lignin composition in xylem cell walls mostly results from random polymerization and is therefore largely determined by the dynamics of monomer biosynthesis in the cytoplasm. ${ }^{2}$ These observations are relevant for biorefinery applications because G-type lignin is a negative predictor of wood saccharification into valuable glucose. ${ }^{18}$ FLIM could help understand the mechanisms underlying the dynamics of Gtype and S-type lignin accumulation to help select or create superior wood biomass feedstocks for biorefinery.

The 11 tree genotypes used in this study were grown under two different nutrition conditions: nutrition with a nitrogen source mostly in an organic form and a more classical nutrition with nitrogen mostly in the mineral form (Dataset S2). These different treatments did not result in consistent changes in the wood chemical composition across the entire tree population, although specific genotypes showed significant differences in accumulation of S-type or H-type lignin (Figure 5c,d). Wildtype trees in particular showed an increased S-type lignin content and an increased S-type to G-type lignin ratio under mineral nitrogen nutrition treatment. Some of these singular differences may arise from the possible occurrence of tension wood, a specific type of wood nearly devoid of lignin, forming in response to external conditions that exert physical strain on the stem. ${ }^{47}$ Alternatively, or in addition, there may be a response to nitrogen nutrition in terms of heterogeneity of lignification. Using FLIM, we observed the developing wood of wild-type trees and also found an increase in the S-type to Gtype lignin ratio under mineral nutrition (Figure $5 \mathrm{~h}, \mathrm{i}$ ). These FLIM observations in situ also revealed that these differences in lignin between treatment occurred specifically in the xylem fiber cell type, but not in vessels (Figure 5h,i), providing an interesting anatomical resolution to the analysis of wood chemical composition between treatments.

\section{CONCLUSIONS}

FLIM has previously been applied to detecting chemical differences in biological samples, ${ }^{26}$ for purposes of classification such as distinguishing pathological versus healthy samples in diagnosis ${ }^{63}$ or such as distinguishing between metabolic states or between differentiation states in living cells. ${ }^{64}$ These strategies, however, do not quantify aspects of the chemical composition of the fluorophores based on FLIM. Our approach using FLIM for chemical characterization of naturally fluorescent lignocellulosic cell walls shows that it is possible to take a significant step further: we provide an example that FLIM can be used for in situ quantitative measurements of the chemical composition of naturally fluorescent molecules, even in vivo. Similar to lignin, other important biological molecules are known for their fluorescence properties. Such molecules include collagen, ${ }^{65}$ keratin and elastin, ${ }^{63} \mathrm{NADH},{ }^{64}$ or resilin in the shell of insects. ${ }^{66}$ These molecules could in theory also be chemically characterized in situ by FLIM, if similar efforts were undertaken for the calibration of statistical models as those we present here for plant lignocellulosic cell walls.

\section{MATERIALS AND METHODS}

Plant material and Growth Conditions. The Arabidopsis thaliana genotypes consisted of Col-0 wild-type; the $c 4 h-3 / r e f 3-3$ which contains a point mutation in the cinnamate-4-hydroxylase enzyme-encoding gene ( $\mathrm{C} 4 \mathrm{H}$; At2g30490) that results in a still partially functional protein; ${ }^{34}$ the previously generated gux1 gux2 double mutant (targeting AT3G18660 and AT4G33330) that lack functional glucuronic acid substitution of xylan proteins; ${ }^{36,37}$ and the irx7/fra8 mutant (targeting At2g28110), which harbors defects in xylan biosynthesis. ${ }^{39}$ These plants were grown for 8 weeks in growth chambers with $16 \mathrm{~h}$ simulated day-light and $8 \mathrm{~h}$ night, with semicontrolled temperatures oscillating between 18 and $23{ }^{\circ} \mathrm{C}$. After these 8 weeks, a $1 \mathrm{~cm}$ long piece of stem was sampled $2 \mathrm{~cm}$ over the soil for further sectioning and FLIM observations. For live imaging by FLIM of the first lignifying protoxylem cells, 4.5 days old (after germination) seedlings of Col- 0 and $c 4 h-3$ were used.

The collection of hybrid aspen (Populus tremula $\mathrm{x}$ tremuloides Michx.) trees used as "standards" consisted of 11 genotypes: the wildtype T89 clone, as well as 10 transgenic lines, targeting six genes known or thought to be linked to wood cell wall properties (Dataset $S 1^{18,67-70}$ ). To generate replicates, the trees were clonally propagated in vitro as previously described. ${ }^{71}$ After 3 weeks of in vitro growth, eight (transgenics) or 10 (wild-type) trees from each genotype were transferred to $1 \mathrm{~L}$ soil pots and grown for 9 weeks under $16 \mathrm{~h} / 8 \mathrm{~h}$ day-night cycles. Following an initial period of 3 weeks of tree establishment under the soil conditions, the trees started receiving fertilization with increasing doses of either one of two nutrient solutions (Dataset S2): the arGrow solution (Arevo AB, Sweden) with nitrogen mostly in the organic form arginine, and SW Bouyant Rika-S (SW Horto AB, Sweden) with nitrogen mostly in the mineral form of nitrate (Dataset S2).

Specific Lignin Samples. The three samples of extracted pine lignin were prepared by dioxane $/ \mathrm{HCl}$ acidolysis following the "reflux method," $" 2$ but with some modifications: pine wood samples were milled to a particle size of $200 \mu \mathrm{m}$ (Retsch ZM1000 centrifugal mill, Retsch GmbH, Germany). The milled samples $(20 \mathrm{~g})$ were placed in $250 \mathrm{~mL}$ dioxane $/ \mathrm{HCl} 2 \mathrm{M}(9: 1 \mathrm{v} / \mathrm{v})$ under a flow of nitrogen gas for 3 h. Another $250 \mathrm{~mL}$ of dioxane/ $\mathrm{HCl} 2 \mathrm{M}$ were added, and the solution was heated to a reflux for $30 \mathrm{~min}$. This solution was filtered and poured onto $8.4 \mathrm{~g}$ of $\mathrm{NaHCO}_{3}$ for acid neutralization. Following the near complete evaporation of the dioxane, the samples were thoroughly washed with large volumes of ultrapure water, centrifuged, and finally air-dried.

The synthesized lignin samples, so-called dehydrogenation polymers (DHPs), were generated in vitro as previously described, ${ }^{73,74}$ but with an equal mix of coniferyl alcohol and sinapyl alcohol to generate a polymer of S-type and G-type lignin units with a 1:1 ratio.

Sampling and Preparation for Different Analyses. After 9 weeks of growth, the 90 trees were harvested (Figure S2). Stem pieces were collected $15 \mathrm{~cm}$ above ground to generate the samples for all the cell wall chemistry and imaging analyses. From these stem pieces, several sections were made: The $9 \mathrm{~cm}$ toward the base of the stem were freeze-dried (CoolSafe Pro 110-4, LaboGene A/S, Denmark) and milled (Retsch ZM200 centrifugal mill), and the resulting wood powder with a particle size of less than $0.5 \mathrm{~mm}$ was collected for subsequent $2 \mathrm{D}-\mathrm{NMR}$ analyses. The next $1 \mathrm{~cm}$ was kept apart, and the following $2 \mathrm{~cm}$ of stem were collected and $100 \mu \mathrm{m}$ sections were generated using a vibratome (Leica VT1000S, Leica Biosystems $\mathrm{GmbH}$, Germany). These sections were mounted in $0.1 \mathrm{M}$ phosphate buffer at $\mathrm{pH} 5.8$ (close to the physiological $\mathrm{pH}$ of plant cell walls) for direct observation by FLIM. The next $4 \mathrm{~cm}$ of stem were freeze-dried, milled, and sieved to separate particles of different sizes. Particles with a diameter between 0.1 and $0.5 \mathrm{~mm}$ were collected for enzymatic saccharification assay. ${ }^{75}$ Particles with diameter lower than $0.1 \mathrm{~mm}$ were collected separately for the analysis of the cell wall lignin content and composition by pyrolysis coupled with GC and MS.

Arabidopsis thaliana stem samples, collected $2 \mathrm{~cm}$ above ground after 8 weeks of plant growth, were cut with a vibratome as described 
above to generate $100 \mu \mathrm{m}$ sections. These sections were also mounted in $0.1 \mathrm{M}$ phosphate buffer at $\mathrm{pH} 5.8$ and observed by FLIM soon after.

Analyses of Wood Cell Wall Chemical Composition and Saccharification. The cell wall content in carbohydrates (indiscriminately cellulose, hemicelluloses, and other possible saccharides), as well as the lignin content and composition, was measured by pyrolysis-GC/MS, as previously described. ${ }^{58}$

Enzymatic saccharification assays, measuring the release of five monosaccharides (glucose xylose, galactose, mannose and arabinose) either without pretreatment or after an acidic pretreatment, were performed on the wood powder of particle size 0.1 to $0.5 \mathrm{~mm}$, as previously described $^{73}$ using a liquid enzyme preparation ( $5 \mathrm{mg}$ Cellic CTec2, obtained from Sigma-Aldrich, St. Louis, MO, USA). The concentrations of the five monosaccharides were determined using HPAEC-PAD (ICS-5000, Dionex, Sunnyvale, CA, USA) following a previously described procedure. ${ }^{76}$ The GPR, which is based on the initial $2 \mathrm{~h}$ of saccharification, was determined as previously described.

Analysis of the relative abundance of covalent bonds within the lignin polymers relied 2D-NMR, as previously described. ${ }^{54}$ These analyses required pooling of samples to gather a sufficient amount of wood powder with a particle size between 0.1 and $0.5 \mathrm{~mm}$ and could only be performed on a subset of trees (Dataset S4).

Imaging and Image Analysis. FLIM images were acquired directly on the sections of tree stems or Arabidopsis thaliana stems, solely based on lignin natural fluorescence. All fluorescence lifetime imaging microscopy observations relied on the time-correlated single photon counting methodology $\left(\mathrm{TCSPC}^{30}\right)$.

The Arabidopsis thaliana stem sections were imaged with a confocal microscope LSM710 (Carl Zeiss Microscopy GmbH, Germany) using a Chameleon TiSa accordable $80 \mathrm{Mhz}$ pulsed laser (Coherent Inc., USA) for excitation and a MW-FLIM detector system with a SPC 150 photocounting card (Becker \& Hickl GmbH, Germany) for detection, as previously described. ${ }^{32}$ Images were processed using SPCImage 6.2 (Becker \& Hickl GmbH, Germany) to extract fluorescence lifetime data specifically from xylem cell walls (Figure 1), selected as regions of interest (ROIs).

All other samples were imaged with a confocal microscope LSM880 (Carl Zeiss Microscopy GmbH, Germany) coupled with a FLIM Upgrade Kit for LSMs (PicoQuant GmbH, Germany), including PicoQuant pulsed diode lasers (tunable laser repetition rate between 2.5 and $40 \mathrm{MHz}$ ) and $\mathrm{HyD}$ single-molecule detectors for photon detection and counting. Tile imaging had to be performed (4 $\times 4$ tiles with $10 \%$ overlap) with a $\times 4$ lens when looking at entire tree stem sections. In addition, images of developing wood areas were also acquired with a $\times 20$ lens for higher resolution.

FLIM parameters were extracted using the SymPhoTime64 software (SPT64, PicoQuant GmbH, Germany). FLIM fitting on the lifetime decay curves revealed that the best fits could be obtained using three exponentials, meaning that the average lifetime could be best described as a composite resulting from three different lifetimes. FLIM parameters (Dataset S3) were extracted on whole sections, allowing comparison with the analytical methods that record the cell wall chemical composition of homogenized ground samples (Dataset S3). FLIM parameters could also be extracted from specific wood areas or specific cell types, using the ROI function of the SPT software, allowing for manual selection of any pixel or group of pixels on the images.

Statistical Modeling. Statistical modeling followed a previously established methodology ${ }^{18,43}$ that consists of using three different types of models to select the most predictive model based on leaveone-out cross validation. Briefly, statistical relationships between FLIM parameters (independent variables) and each wood cell wall or saccharification trait (dependent variable) were explored by multiple linear regression, as well as by machine learning algorithms known as $\mathrm{GAMs}^{49}$ and random forests. ${ }^{50}$

All types of models were implemented in $\mathrm{R}$, using the package "mgcv" for $\mathrm{GAMs}^{78}$ and the package "ranger" for random forests. For each dependent variable, at least 15 models from each type (i.e., at least 45 models) were generated. Model validation consisted of leaving out one observation (one tree), regenerating the model based on the remaining 89 other trees, and predicting the value of the one tree that had been left out. This procedure was repeated until each observation had been left out, and the difference between the predictions and the actual values for each tree allows calculating the Q2 (with Q2 between 1 and minus infinity, 1 indicating a perfect prediction).

\section{ASSOCIATED CONTENT}

\section{st Supporting Information}

The Supporting Information is available free of charge at https://pubs.acs.org/doi/10.1021/acssuschemeng.1c06780.

Figure S1: Compilation of FLIM images of entire wood sections for all 90 trees and Figure S2: Workflow of material collection and processing (PDF)

Dataset S1: List of tree genotypes used in this study (XLSX)

Dataset S2: Details of tree nutrition treatments' composition (XLSX)

Dataset S3: Tables with all the measurements performed in this study (XLSX)

Dataset S4: Summaries of the most predictive statistical models for each trait of interest (XLSX)

\section{AUTHOR INFORMATION}

\section{Corresponding Authors}

Sacha Escamez - Umeå Plant Science Centre (UPSC), Department of Forest Genetics and Plant Physiology, Swedish University of Agricultural Sciences, Umeå SE-901 83, Sweden; Umeå Plant Science Centre (UPSC), Department of Plant Physiology, Umeå University, Umeå SE-901 87, Sweden; (1) orcid.org/0000-0001-7049-6978; Email: sacha.escamez@slu.se, sacha.escamez.phd@ gmail.com

Gabriel Paës - Université de Reims Champagne Ardenne, INRAE, FARE, Reims 51100, France; (1) orcid.org/00000003-0239-9716; Email: gabriel.paes@inrae.fr

\section{Authors}

Christine Terryn - PICT platform, Université de Reims Champagne Ardenne, Reims 51100, France

Madhavi Latha Gandla - Department of Chemistry, Umeå University, Umeå SE-901 87, Sweden

Zakiya Yassin - RISE AB, Stockholm SE-114 28, Sweden

Gerhard Scheepers - RISE AB, Stockholm SE-114 28, Sweden

Torgny Näsholm - Umeå Plant Science Centre (UPSC), Department of Forest Genetics and Plant Physiology and Department of Forest Ecology and Management, Swedish University of Agricultural Sciences, Umeå SE-901 83, Sweden

Ola Sundman - Department of Chemistry, Umeå University, Umeå SE-901 87, Sweden

Leif J. Jönsson - Department of Chemistry, Umeå University, Umeå SE-901 87, Sweden; 다이.org/0000-0003-38660111

Judith Lundberg-Felten - Umeå Plant Science Centre (UPSC), Department of Forest Genetics and Plant Physiology, Swedish University of Agricultural Sciences, Umeå SE-901 83, Sweden

Hannele Tuominen - Umeå Plant Science Centre (UPSC), Department of Forest Genetics and Plant Physiology, Swedish University of Agricultural Sciences, Umeå SE-901 83, Sweden 
Totte Niittylä - Umeå Plant Science Centre (UPSC),

Department of Forest Genetics and Plant Physiology, Swedish

University of Agricultural Sciences, Umeå SE-901 83, Sweden

Complete contact information is available at:

https://pubs.acs.org/10.1021/acssuschemeng.1c06780

\section{Author Contributions}

S.E. and G.P. designed the study, with conceptual contributions and feedback from C.T., G.S., T.N., O.S., L.J.J., J.L.-F., H.T., and T.N. S.E., T.N., J.L.-F., and H.T. contributed with the design and production of genetically engineered trees used in this study. S.E., C.T., M.L.G., and Z.Y. performed the experiments: S.E. grew the plants, harvested the samples, and prepared the stem sections. C.T. and S.E. performed FLIM imaging of Arabidopsis thaliana stems. S.E. performed FLIM imaging of wood stems and synthesized and extracted lignins, as well as live imaging of lignin deposition dynamics in Arabidopsis thaliana seedlings. S.E., M.L.G., and Z.Y. prepared the samples for all other analytical methods. M.L.G. performed the saccharification assays (including pretreatments when relevant). S.E. extracted data (lifetime parameters) from FLIM analyses and performed statistical analyses and modeling. S.E. and G.P. wrote the manuscript, with help from all the coauthors.

\section{Notes}

The authors declare no competing financial interest.

\section{ACKNOWLEDGMENTS}

The authors wish to acknowledge the valuable technical help from Junko Takahashi-Schmidt (pyrolysis-GC/MS, UPSC biopolymer analytical platform), Anouck Habrant (synthesis of DHP, FARE lab), and David Crônier (2D NMR analysis, FARE lab). This work was funded by the Swedish Strategic Research Environment Bio4Energy (https://www.bio4energy. se) through two grants: the Bio4Energy Special Call Grant 2018 and the Bio4Energy Strategic Funds Grant 2019. Bio4Energy is one of three strategic research environments funded by the Swedish Energy Agency.

\section{REFERENCES}

(1) Bar-On, Y. M.; Phillips, R.; Milo, R. The biomass distribution on Earth. Proc. Natl. Acad. Sci. U. S. A. 2018, 115, 6506-6511.

(2) Boerjan, W.; Ralph, J.; Baucher, M. Lignin biosynthesis. Annu. Rev. Plant Biol. 2003, 54, 519-546.

(3) Somerville, C. Cellulose synthesis in higher plants. Annu. Rev. Cell Dev. Biol. 2006, 22, 53-78.

(4) Scheller, H. V.; Ulvskov, P. Hemicelluloses. Annu. Rev. Plant Biol. 2010, 61, 263-289.

(5) Meents, M. J.; Watanabe, Y.; Samuels, A. L. The cell biology of secondary cell wall biosynthesis. Ann. Bot. 2018, 121, 1107-1125.

(6) Hosmani, P. S.; Kamiya, T.; Danku, J.; Naseer, S.; Geldner, N.; Guerinot, M. L.; Salt, D. E. Dirigent domain-containing protein is part of the machinery required for formation of the lignin-based Casparian strip in the root. Proc. Natl. Acad. Sci. U. S. A. 2013, 110, 1449814503.

(7) Lee, Y.; Rubio, M. C.; Alassimone, J.; Geldner, N. A mechanism for localized lignin deposition in the endodermis. Cell 2013, 153, 402-412.

(8) Lee, Y.; Yoon, T. H.; Lee, J.; Jeon, S. Y.; Lee, J. H.; Lee, M. K.; Chen, H.; Yun, J.; Oh, S. Y.; Wen, X.; Cho, H. K.; Mang, H.; Kwak, J. M. A Lignin Molecular Brace Controls Precision Processing of Cell Walls Critical for Surface Integrity in Arabidopsis. Cell 2018, 173, 1468-1480.e9.
(9) Lee, M. H.; Jeon, H. S.; Kim, S. H.; Chung, J. H.; Roppolo, D.; Lee, H. J.; Cho, H. J.; Tobimatsu, Y.; Ralph, J.; Park, O. K. Ligninbased barrier restricts pathogens to the infection site and confers resistance in plants. EMBO J. 2019, 38, No. e101948.

(10) Reyt, G.; Chao, Z.; Flis, P.; Salas-González, I.; Castrillo, G.; Chao, D. Y.; Salt, D. E. Uclacyanin proteins are required for lignified nanodomain formation within casparian strips. Curr. Biol. 2020, 30, 4103-4111.e6.

(11) Reyt, G.; Ramakrishna, P.; Salas-González, I.; Fujita, S.; Love, A.; Tiemessen, D.; Lapierre, C.; Morreel, K.; Calvo-Polanco, M.; Flis, P.; Geldner, N.; Boursiac, Y.; Boerjan, W.; George, M. W.; Castrillo, G.; Salt, D. E. Two chemically distinct root lignin barriers control solute and water balance. Nat. Commun. 2021, 12, 2320.

(12) Jones, L.; Ennos, A. R.; Turner, S. R. Cloning and characterization of irregular xylem4 (irx4): a severely lignin-deficient mutant of Arabidopsis. Plant J. 2001, 26, 205-216.

(13) Weng, J. K.; Mo, H.; Chapple, C. Over-expression of F5H in COMT-deficient Arabidopsis leads to enrichment of an unusual lignin and disruption of pollen wall formation. Plant J. 2010, 64, 898-911.

(14) Hao, Z.; Avci, U.; Tan, L.; Zhu, X.; Glushka, J.; Pattathil, S.; Eberhard, S.; Sholes, T.; Rothstein, G. E.; Lukowitz, W.; Orlando, R.; Hahn, M. G.; Mohnen, D. Loss of Arabidopsis GAUT12/IRX8 causes anther indehiscence and leads to reduced G lignin associated with altered matrix polysaccharide deposition. Front. Plant Sci. 2014, 5, 121.

(15) Percival Zhang, Y.-H. Next generation biorefineries will solve the food, biofuels, and environmental trilemma in the energy-foodwater nexus. Energy Sci. Eng. 2013, 1, 27-41.

(16) Ragauskas, A. J.; Beckham, G. T.; Biddy, M. J.; Chandra, R.; Chen, F.; Davis, M. F.; Davison, B. H.; Dixon, R. A.; Gilna, P.; Keller, M.; Langan, P.; Naskar, A. K.; Saddler, J. N.; Tschaplinski, T. J.; Tuskan, G. A.; Wyman, C. Lignin valorization: improving lignin processing in the biorefinery. Science 2014, 344, 1-10.

(17) McCann, M. C.; Carpita, N. C. Biomass recalcitrance: a multiscale, multi-factor and conversion-specific property. J. Exp. Bot. 2015, 66, 4109-4118.

(18) Escamez, S.; Latha Gandla, M.; Derba-Maceluch, M.; Lundqvist, S. O.; Mellerowicz, E. J.; Jönsson, L. J.; Tuominen, H. \& Tuominen, H.A collection of genetically engineered Populus trees reveals wood biomass traits that predict glucose yield from enzymatic hydrolysis. Sci. Rep. 2017, 7, 15798.

(19) Zoghlami, A.; Paës, G. Lignocellulosic biomass: understanding recalcitrance and predicting hydrolysis. Front. Chem. 2019, 7, 874.

(20) Ralph, J.; Lapierre, C.; Boerjan, W. Lignin structure and its engineering. Curr. Opin. Biotechnol. 2019, 56, 240-249.

(21) Ménard, D.; Blaschek, L.; Kriechbaum, K.; Lee, C.C.; Zhu, C.; Bacsik, Z.; Bergström, L.; Mathew, A.; Kajita, S.; Pesquet, E. Specific and dynamic lignification at the cell-type level controls plant physiology and adaptability. bioRxiv, 2021.

(22) Donaldson, L. A.; Radotic, K. Fluorescence lifetime imaging of lignin autofluorescence in normal and compression wood. J. Microsc. 2013, 251, 178-187.

(23) Simon, C.; Lion, C.; Biot, C.; Gierlinger, N.; Hawkins, S. Lignification and advances in lignin imaging in plant cell walls. Ann. Plant Rev. Online 2018, 909-940.

(24) Donaldson, L. Autofluorescence in plants. Molecules 2020, 25, $1-20$.

(25) Decou, R.; Serk, H.; Ménard, D.; Pesquet, E. in Xylem; Springer, 2017), 233-247.

(26) Berezin, M. Y.; Achilefu, S. Fluorescence lifetime measurements and biological imaging. Chem. Rev. 2010, 110, 2641-2684.

(27) Einstein, A. The Quantum Theory of Radiation. Phys. Z. 1917, $18,121-128$.

(28) Strickler, S. J.; Berg, R. A. Relationship between absorption intensity and fluorescence lifetime of molecules. J. Chem. Phys. 1962, 37, 814-822.

(29) Wang, X. F.; Periasamy, A.; Herman, B.; Coleman, D. M. Fluorescence lifetime imaging microscopy (FLIM): instrumentation and applications. Crit. Rev. Anal. Chem. 1992, 23, 369-395. 
(30) Becker, W. Advanced time-correlated single photon counting techniques; (Springer Science \& Business Media, 81, 1-400, 2005).

(31) Wightman, R.; Busse-Wicher, M.; Dupree, P. Correlative FLIM-confocal-Raman mapping applied to plant lignin composition and autofluorescence. Micron 2019, 126, No. 102733.

(32) Auxenfans, T.; Terryn, C.; Paës, G. Seeing biomass recalcitrance through fluorescence. Sci. Rep. 2017, 7, 8838.

(33) Chabbert, B.; Terryn, C.; Herbaut, M.; Vaidya, A.; Habrant, A.; Paës, G.; Donaldson, L. Fluorescence techniques can reveal cell wall organization and predict saccharification in pretreated wood biomass. Ind. Crops Prod. 2018, 123, 84-92.

(34) Schilmiller, A. L.; Stout, J.; Weng, J. K.; Humphreys, J.; Ruegger, M. O.; Chapple, C. Mutations in the cinnamate 4hydroxylase gene impact metabolism, growth and development in Arabidopsis. Plant J. 2009, 60, 771-782.

(35) Van Acker, R.; Vanholme, R.; Storme, V.; Mortimer, J. C.; Dupree, P.; Boerjan, W. Lignin biosynthesis perturbations affect secondary cell wall composition and saccharification yield in Arabidopsis thaliana. Biotechnol. Biofuels 2013, 6, 46.

(36) Mortimer, J. C.; Miles, G. P.; Brown, D. M.; Zhang, Z.; Segura, M. P.; Weimar, T.; Yu, X.; Seffen, K. A.; Stephens, E.; Turner, S. R.; Dupree, P. Absence of branches from xylan in Arabidopsis gux mutants reveals potential for simplification of lignocellulosic biomass. Proc. Natl. Acad. Sci. U. S. A. 2010, 107, 17409-17414.

(37) Bromley, J. R.; Busse-Wicher, M.; Tryfona, T.; Mortimer, J. C.; Zhang, Z.; Brown, D. M.; Dupree, P. GUX 1 and GUX 2 glucuronyltransferases decorate distinct domains of glucuronoxylan with different substitution patterns. Plant J. 2013, 74, 423-434.

(38) Watanabe, T.; Koshijima, T. Evidence for an ester linkage between lignin and glucuronic acid in lignin-carbohydrate complexes by DDQ-oxidation. Agric. Biol. Chem. 1988, 52, 2953-2955.

(39) Zhong, R.; Peña, M. J.; Zhou, G. K.; Nairn, C. J.; Wood-Jones, A.; Richardson, E. A.; Morrison, W. H., III; Darvill, A. G.; York, W. S.; Ye, Z. H. Arabidopsis fragile fiber8, which encodes a putative glucuronyltransferase, is essential for normal secondary wall synthesis. Plant Cell 2005, 17, 3390-3408.

(40) Jansson, S., Bhalerao, R., Groover, A. Genetics and genomics of Populus; (Springer, 8, 2010).

(41) Tuskan, G. A.; et al. The genome of black cottonwood, Populus trichocarpa (Torr. \& Gray). Science 2006, 313, 1596-1604.

(42) Lin, Y.-C.; Wang, J.; Delhomme, N.; Schiffthaler, B.; Sundström, G.; Zuccolo, A.; Nystedt, B.; Hvidsten, T. R.; de la Torre, A.; Cossu, R. M.; Hoeppner, M. P.; Lantz, H.; Scofield, D. G.; Zamani, N.; Johansson, A.; Mannapperuma, C.; Robinson, K. M.; Mähler, N.; Leitch, I. J.; Pellicer, J.; Park, E. J.; van Montagu, M.; van de Peer, Y.; Grabherr, M.; Jansson, S.; Ingvarsson, P. K.; Street, N. R. Functional and evolutionary genomic inferences in Populus through genome and population sequencing of American and European aspen. Proc. Natl. Acad. Sci. U. S. A. 2018, 115, E10970-E10978.

(43) Escamez, S.; Luomaranta, M.; Mähler, N.; Gandla, M.L.; Robinson, K.M.; Yassin, Z.; Grahn, T.; Scheepers, G.; Stener, L.G.; Jönsson, L.J.; Jansson, S.; Street, N.; Tuominen, H. Identification of genetic markers and wood properties that predict wood biorefinery potential in aspen bioenergy feedstock (Populus tremula). bioRxiv, 2021.

(44) Dickmann, D. I. Silviculture and biology of short-rotation woody crops in temperate regions: Then and now. Biomass Bioenergy 2006, 30, 696-705.

(45) Mola-Yudego, B.; Arevalo, J.; Díaz-Yáñez, O.; Dimitriou, I.; Haapala, A.; Carlos Ferraz Filho, A.; Selkimäki, M.; Valbuena, R. Wood biomass potentials for energy in northern Europe: Forest or plantations? Biomass Bioenergy 2017, 106, 95-103.

(46) Euring, D.; Löfke, C.; Teichmann, T.; Polle, A. Nitrogen fertilization has differential effects on $\mathrm{N}$ allocation and lignin in two Populus species with contrasting ecology. Trees 2012, 26, 1933-1942.

(47) Pitre, F. E.; Pollet, B.; Lafarguette, F.; Cooke, J. E.; MacKay, J. J.; Lapierre, C. Effects of increased nitrogen supply on the lignification of poplar wood. J. Agric. Food Chem. 2007, 55, 10306-10314.

(48) Franklin, O.; Näsholm, T.; Högberg, P.; Högberg, M. N. Forests trapped in nitrogen limitation-an ecological market perspective on ectomycorrhizal symbiosis. New Phytol. 2014, 203, 657-666.

(49) Hastie, T.; Tibshirani, R. Generalized additive models. Stat. Sci. 1986, 1, 297-310.

(50) Breiman, L. Random forests. Mach. Learn. 2001, 45, 5-32.

(51) Wang, J. P.; Matthews, M. L.; Williams, C. M.; Shi, R.; Yang, C.; Tunlaya-Anukit, S.; Chen, H. C.; Li, Q.; Liu, J.; Lin, C. Y.; Naik, P.; Sun, Y. H.; Loziuk, P. L.; Yeh, T. F.; Kim, H.; Gjersing, E.; Shollenberger, T.; Shuford, C. M.; Song, J.; Miller, Z.; Huang, Y. Y.; Edmunds, C. W.; Liu, B.; Sun, Y.; Lin, Y. C. J.; Li, W.; Chen, H.; Peszlen, I.; Ducoste, J. J.; Ralph, J.; Chang, H. M.; Muddiman, D. C.; Davis, M. F.; Smith, C.; Isik, F.; Sederoff, R.; Chiang, V. L. Improving wood properties for wood utilization through multi-omics integration in lignin biosynthesis. Nat. Commun. 2018, 9, 1579.

(52) Wang, J. P.; Naik, P. P.; Chen, H. C.; Shi, R.; Lin, C. Y.; Liu, J.; Shuford, C. M.; Li, Q.; Sun, Y. H.; Tunlaya-Anukit, S.; Williams, C. M.; Muddiman, D. C.; Ducoste, J. J.; Sederoff, R. R.; Chiang, V. L.; Gjersing, E.; Shollenberger, T.; Shuford, C. M.; Song, J.; Miller, Z.; Huang, Y.-Y.; Edmunds, C. W.; Liu, B.; Sun, Y.; Lin, Y.-C. J.; Li, W.; Chen, H.; Peszlen, I.; Ducoste, J. J.; Ralph, J.; Chang, H.-M.; Muddiman, D. C.; Davis, M. F.; Smith, C.; Isik, F.; Sederoff, R.; Chiang, V. L. Complete proteomic-based enzyme reaction and inhibition kinetics reveal how monolignol biosynthetic enzyme families affect metabolic flux and lignin in Populus trichocarpa. Plant Cell 2014, 26, 894-914.

(53) Morreel, K.; Dima, O.; Kim, H.; Lu, F.; Niculaes, C.; Vanholme, R.; Dauwe, R.; Goeminne, G.; Inzé, D.; Messens, E.; Ralph, J.; Boerjan, W. Mass spectrometry-based sequencing of lignin oligomers. Plant Physiol. 2010, 153, 1464-1478.

(54) Auxenfans, T.; Crônier, D.; Chabbert, B.; Paës, G. Understanding the structural and chemical changes of plant biomass following steam explosion pretreatment. Biotechnol. Biofuels 2017, 10, 36.

(55) Alejandro, S.; Lee, Y.; Tohge, T.; Sudre, D.; Osorio, S.; Park, J.; Bovet, L.; Lee, Y.; Geldner, N.; Fernie, A. R.; Martinoia, E. AtABCG29 is a monolignol transporter involved in lignin biosynthesis. Curr. Biol. 2012, 22, 1207-1212.

(56) Naseer, S.; Lee, Y.; Lapierre, C.; Franke, R.; Nawrath, C.; Geldner, N. Casparian strip diffusion barrier in Arabidopsis is made of a lignin polymer without suberin. Proc. Natl. Acad. Sci. U. S. A. 2012, 109, 10101-10106.

(57) Terashima, N.; Fukushima, K. in Plant Cell Wall Polymers (Vol. 399 ACS Symposium Series), Ch. 11; American Chemical Society, 1989), $160-168$.

(58) Gerber, L.; Öhman, D.; Kumar, M.; Ranocha, P.; Goffner, D.; Sundberg, B. High-throughput microanalysis of large lignocellulosic sample sets by pyrolysis-gas chromatography/mass spectrometry. Physiol. Plant. 2016, 156, 127-138.

(59) Terashima, N. A new mechanism for formation of a structurally ordered protolignin macromolecule in the cell wall of tree xylem. J. Pulp Pap. Sci. 1990, 16, J150-J155.

(60) Marita, J. M.; Ralph, J.; Hatfield, R. D.; Chapple, C. NMR characterization of lignins in Arabidopsis altered in the activity of ferulate 5-hydroxylase. Proc. Natl. Acad. Sci. U. S. A. 1999, 96, 1232812332 .

(61) Blaschek, L.; Champagne, A.; Dimotakis, C.; Nuoendagula; Decou, R.; Hishiyama, S.; Kratzer, S.; Kajita, S.; Pesquet, E. Cellular and genetic regulation of coniferaldehyde incorporation in lignin of herbaceous and woody plants by quantitative wiesner staining. Front. Plant Sci. 2020, 11, 1-17.

(62) Zhao, Y.; Yu, X.; Lam, P. Y.; Zhang, K.; Tobimatsu, Y.; Liu, C. J. Monolignol acyltransferase for lignin p-hydroxybenzoylation in Populus. Nat. Plants 2021, 7, 1288-1300.

(63) Galletly, N. P.; McGinty, J.; Dunsby, C.; Teixeira, F.; RequejoIsidro, J.; Munro, I.; Elson, D. S.; Neil, M. A. A.; Chu, A. C.; French, P. M. W.; Stamp, G. W. Fluorescence lifetime imaging distinguishes basal cell carcinoma from surrounding uninvolved skin. Brit. J. Dermatol. 2008, 159, 152-161. 
(64) Stringari, C.; Cinquin, A.; Cinquin, O.; Digman, M. A.; Donovan, P. J.; Gratton, E. Phasor approach to fluorescence lifetime microscopy distinguishes different metabolic states of germ cells in a live tissue. Proc. Natl. Acad. Sci. U. S. A. 2011, 108, 13582-13587.

(65) Sherlock, B. E.; Harvestine, J. N.; Mitra, D.; Haudenschild, A.; $\mathrm{Hu}$, J.; Athanasiou, K. A.; Leach, J. K.; Marcu, L. Nondestructive assessment of collagen hydrogel cross-linking using time-resolved autofluorescence imaging. J. Biomed. Opt. 2018, 23, 1-9.

(66) Reinhardt, K.; Breunig, H. G.; König, K. Autofluorescence lifetime variation in the cuticle of the bedbug Cimex lectularius. Arthropod Struct. Dev. 2017, 46, 56-62.

(67) Gerber, L.; Zhang, B.; Roach, M.; Rende, U.; Gorzsás, A.; Kumar, M.; Burgert, I.; Niittylä, T.; Sundberg, B. Deficient sucrose synthase activity in developing wood does not specifically affect cellulose biosynthesis, but causes an overall decrease in cell wall polymers. New Phytol. 2014, 203, 1220-1230.

(68) Bünder, A.; Sundman, O.; Mahboubi, A.; Persson, S.; Mansfield, S. D.; Rüggeberg, M.; Niittylä, T. CELLULOSE SYNTHASE INTERACTING 1 is required for wood mechanics and leaf morphology in aspen. Plant J. 2020, 103, 1858-1868.

(69) Seyfferth, C.; Wessels, B. A.; Vahala, J.; Kangasjärvi, J.; Delhomme, N.; Hvidsten, T. R.; Tuominen, H.; Lundberg-Felten, J. Populus ERF85 balances xylem cell expansion and secondary cell wall formation in hybrid aspen. Cell 2021, 10, 1971.

(70) Vahala, J.; Felten, J.; Love, J.; Gorzsás, A.; Gerber, L.; Lamminmäki, A.; Kangasjärvi, J.; Sundberg, B. A genome-wide screen for ethylene-induced Ethylene Response Factors (ERFs) in hybrid aspen stem identifies ERF genes that modify stem growth and wood properties. New Phytol. 2013, 200, 511-522.

(71) Gray-Mitsumune, M.; Blomquist, K.; McQueen-Mason, S.; Teeri, T. T.; Sundberg, B.; Mellerowicz, E. J. Ectopic expression of a wood-abundant expansin PttEXPA1 promotes cell expansion in primary and secondary tissues in aspen. Plant Biotechnol. J. 2008, 6, $62-72$

(72) Monties, B. Preparation of dioxane lignin fractions by acidolysis. Methods Enzymol. 1988, 161, 31-35.

(73) Boukari, I.; Putaux, J. L.; Cathala, B.; Barakat, A.; Saake, B.; Rémond, C.; O’Donohue, M.; Chabbert, B. In vitro model assemblies to study the impact of lignin- carbohydrate interactions on the enzymatic conversion of xylan. Biomacromolecules 2009, 10, 24892498.

(74) Muraille, L.; Pernes, M.; Habrant, A.; Serimaa, R.; Molinari, M.; Aguié-Béghin, V.; Chabbert, B. Impact of lignin on water sorption properties of bioinspired self-assemblies of lignocellulosic polymers. Eur. Polym. J. 2015, 64, 21-35.

(75) Gandla, M. L.; Mähler, N.; Escamez, S.; Skotare, T.; Obudulu, O.; Möller, L.; Abreu, I. N.; Bygdell, J.; Hertzberg, M.; Hvidsten, T. R.; Moritz, T.; Wingsle, G.; Trygg, J.; Tuominen, H.; Jönsson, L. J. Overexpression of vesicle-associated membrane protein PttVAP27-17 as a tool to improve biomass production and the overall saccharification yields in Populus trees. Biotechnol. Biofuels 2021, 14, 43.

(76) Wang, Z.; Winestrand, S.; Gillgren, T.; Jönsson, L. J. Chemical and structural factors influencing enzymatic saccharification of wood from aspen, birch and spruce. Biomass Bioenergy 2018, 109, 125-134.

(77) Latha Gandla, M.; Derba-Maceluch, M.; Liu, X.; Gerber, L.; Master, E. R.; Mellerowicz, E. J.; Jönsson, L. J. Expression of a fungal glucuronoyl esterase in Populus: Effects on wood properties and saccharification efficiency. Phytochemistry 2015, 112, 210-220.

(78) Wood, S. N. mgcv: GAMs and generalized ridge regression for R. R News 2001, 1, 20-25.

(79) Wright, M.N.; Ziegler, A. ranger: A fast implementation of random forests for high dimensional data in $C++$ and $R$. arXiv, 2015, arXiv: 1508.04409 .

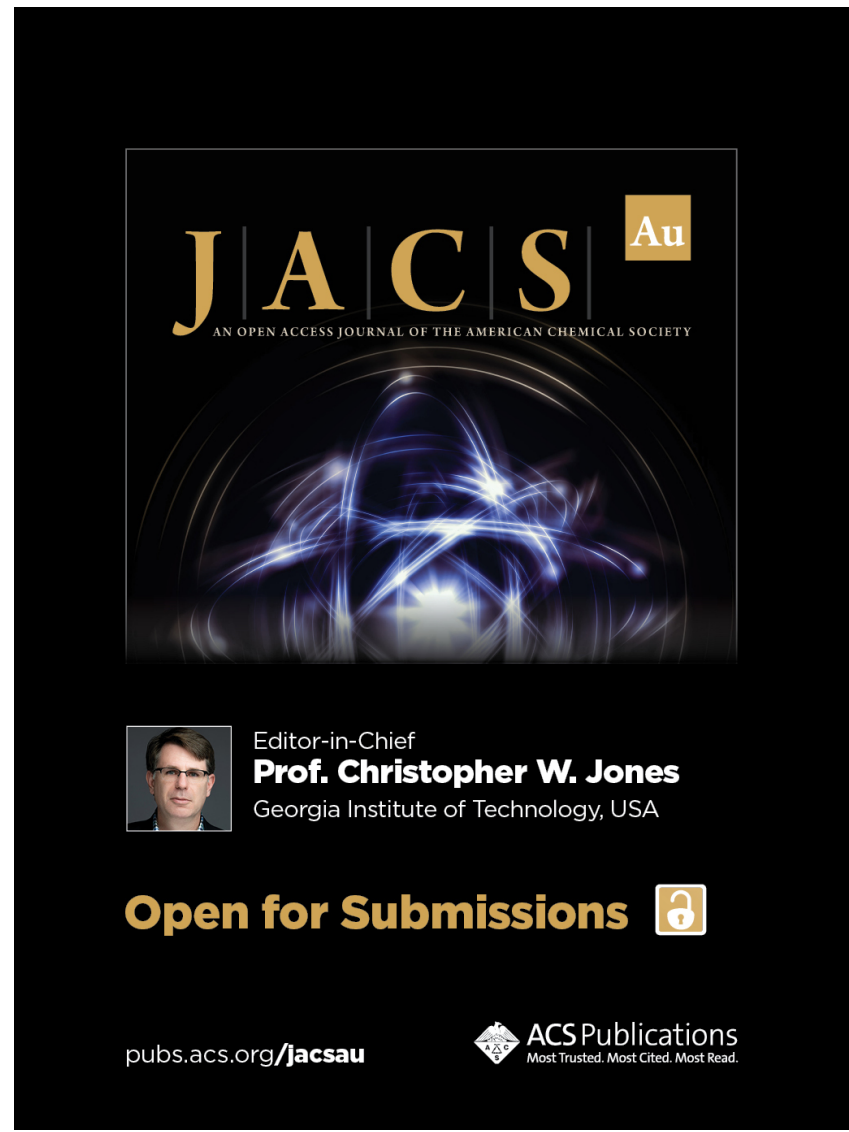

